\title{
Genesis
}

Manuscrits - Recherche - Invention

$51 \mid 2020$

Intertextualité - Exogenèse

\section{Écrire une série TV de Florent Favard et autres publications consacrées à l'écriture scénaristique : à propos d'une tendance à l'oblitération de la dimension génétique}

Alain Boillat

\section{OpenEdition}

\section{Journals}

Édition électronique

URL : https://journals.openedition.org/genesis/5583

DOI : 10.4000/genesis.5583

ISSN : 2268-1590

Éditeur :

Presses universitaires de Paris Sorbonne (PUPS), Société internationale de génétique artistique littéraire et scientifique (SIGALES)

\section{Édition imprimée}

Date de publication : 15 décembre 2020

Pagination : 223-231

ISBN : 979-10-231-0704-3

ISSN : 1167-5101

\section{Référence électronique}

Alain Boillat, «Écrire une série TV de Florent Favard et autres publications consacrées à l'écriture scénaristique : à propos d'une tendance à l'oblitération de la dimension génétique », Genesis [En ligne], 51 | 2020, mis en ligne le 10 janvier 2021, consulté le 07 février 2022. URL : http:// journals.openedition.org/genesis/5583; DOI : https://doi.org/10.4000/genesis.5583 


\section{Écrire une série TV de Florent Favard et autres publications consacrées à l'écriture scénaristique : à propos d'une tendance à l'oblitération de la dimension génétique}

Alain Boillat

$\grave{A}^{\prime}$ l'occasion de la parution, dans la collection «Serial» des Presses universitaires François-Rabelais, d'un ouvrage signé Florent Favard dont le titre Écrire une série TV. La promesse d'un dénouement ${ }^{1}$ peut laisser présager une approche d'obédience génétique susceptible d'intéresser la présente revue, nous proposons de nous interroger de manière plus générale sur la place de ladite approche dans le champ des études cinématographiques ainsi que dans les écrits consacrés au cinéma ou, à l'instar de l'étude de Favard, aux séries télévisuelles. Ces dernières suscitent aujourd'hui un engouement considérable et leur succès, décuplé par les commentaires de blogueurs et les pratiques de consommation qui se sont généralisées à l'ère numérique, a contribué, dans le cas spécifique de la sérialité, à une remise à l'honneur de la narratologie auprès du grand public et dans le domaine académique (alors que les études sur le cinéma l'avaient tendanciellement désertée depuis deux décennies). L'infinitif du titre de Favard pourrait laisser croire de prime abord que l'ouvrage s'inscrit dans la lignée de la kyrielle de publications relevant de l'analyse des récits filmiques (dont Écrire un scénario de Michel Chion, sur lequel nous reviendrons ${ }^{2}$ ) ou de manuels de scénario (à l'instar de L'Écriture de la fiction télévisuelle 3 ) qui se donnent pour tâche de livrer des recettes permettant d'apprendre comment écrire (un «bon scénario»). Ces manuels se caractérisent par une optique normative, ainsi que par une attention majoritairement accordée à la structure en actes, aux modalités de clôture, à la vraisemblance et à la construction des personnages 4 . Les manuels qui font florès à partir des années 1970 - on pense en particulier aux nombreuses rééditions actualisées de l'ouvrage de Syd Field 5 dans le prolongement du New Hollywood qui ravive l'imaginaire de «l'usine à rêves» (et à dollars) ${ }^{6}$ - se présentent comme une sorte de recyclage de l'approche structuraliste, mais sous une forme tellement vulgarisée qu'ils en viennent plus à ressembler à des ouvrages de développement personnel (où le storytelling a aussi son rôle à jouer) qu'à des essais sur le récit. L'étroitesse théorique

1. Florent Favard, Écrire une série TV. La promesse d'un dénouement, Tours, Presses universitaires François-Rabelais, 2019.

2. Michel Chion, Écrire un scénario, Paris/Bry-sur-Marne, Cahiers du cinéma/INA, 1985.

3. Antoine Cucca et Paolo Foti, L'Écriture de la fiction télévisuelle, Paris, Dujarric, 1999.

4. Nous renvoyons à ce propos à notre article «À la recherche du scénario. Le scénario comme objet (d'étude) et pratique (d'écriture)» (Archipel, no 34, décembre 2011, en particulier p. 31-37), où nous mentionnons par opposition l'approche non normative de Raoul Ruiz («Théorie du conflit central», dans Poétique du cinéma, t. I, Paris, Dis Voir, 1995, p. 9-23).

5. L'introduction de l'ouvrage de Syd Field, près de trente ans après la parution originale, est symptomatique à la fois de la place que l'auteur croit occuper dans l'histoire des écrits sur le récit filmique et du contexte éditorial dans lequel le volume a vu le jour : «Quand j’ [en] ai écrit la première version [...], les ouvrages sur l'art et la manière d'écrire un scénario étaient encore rares sur le marché. [...] Il n'existait pas encore vraiment à l'époque de véritable distinction entre écrire pour la scène et écrire pour le cinéma. Scénario a tout changé. Cet ouvrage a posé les principes d'une structure dramatique, établissant ainsi les bases de la narration cinématographique. Ce fût [sic !] aussi le premier à utiliser des films connus et populaires comme exemples, pour illustrer l'art et la manière d'écrire pour le cinéma. [...] À sa première parution, le livre a immédiatement été un best-seller [...]. Ce succès astronomique a visiblement surpris tout le monde. Tout le monde, sauf moi. Au cours de mes enseignements et conférences sur la narration cinématographique au Sheerwood Oaks Experimental College d'Hollywood dans les années 1970, j’ai vu des gens de tous horizons consumés par le désir d'écrire des scénarii. » (Syd Field, Scénario : les bases de la narration cinématographique, Paris, Dixit, 2008 [1979], p. 5). On notera que Field utilise, dans son manuel, des exemples de films, à l'exclusion presque systématique de scénarios de films.

6. À propos des films de cette période, voir Kristin Thompson, Storytelling in the New Hollywood. Understanding Classical Narrative Technique, Cambridge/London, Harvard Univ. Press, 1999. En fait, l'apparition de tels manuels est contemporaine de celle d'Hollywood au début des années 1910 
résultant de la visée prescriptive ${ }^{7}$, l'occultation d'une tradition critique - en général, seul Aristote est cité 8 , tandis que Propp, Barthes ou Greimas sont pillés sans référence aucune - et l'absence d'analyse des exemples convoqués qui sont le lot des manuels de scénario ne concernent toutefois en rien l'ouvrage académique de Favard, qui se propose d'élaborer un cadre théorique rigoureux à partir des acquis récents d'une narratologie qui, s'autodésignant comme «post-classique», entend entre autres s'écarter des approches «immanentes» postulant la clôture du texte ${ }^{9}$ (et même des approches qui intègrent le travail de configuration narrative du lecteur au niveau de la macrostructure) pour investiguer des phénomènes d'émergence ou de transmédialité. Ainsi Favard se montre-t-il toujours soucieux de construire des modèles en exploitant de manière productive la littérature préalable, de filer à travers le livre l'étude de séries-clés dont l'examen se voit ainsi approfondi sur un mode lui-même agréablement «épisodique» et de nuancer ou complexifier l'approche, notamment en envisageant les phénomènes de manière graduelle (la bipolarité entre l'épisodique et le feuilletonnant) et stratifiée (l'ampleur variable d'un arc narratif donné au-delà des unités que sont le pilote, l'épisode et la saison, les jeux de hiérarchisation/reconfiguration des rapports entre lignes principales et secondaires de l'intrigue ou les mouvements délimités à l'échelle de l'intégralité de la série par des points de basculement). L'ouvrage de Favard n'est donc aucunement un manuel. Cependant, l'éditeur d'Écrire une série $T V$ n'a sans doute pas voulu manquer une opportunité de titiller l'inspiration créatrice de son lectorat ${ }^{10}$ en nourrissant une certaine ambiguitté (quelque peu regrettable) que le sous-titre vient toutefois lever : c'est en fait dans la «promesse d'un dénouement» sans cesse différé dans les séries à dominante feuilletonnante que réside l'objet de Favard, et plus précisément dans la promesse plus que dans le dénouement proprement dit - par opposition aux explications des manuels quant aux bonnes manières de clore un récit. L'intérêt de cette approche tient à la spécificité de l'écriture de la plupart des fictions télévisuelles (Favard montre par contraste combien Babylon 5 est une exception), dont l'issue «du» récit (ou d'une pluralité de récits) n'est souvent guère anticipée par les scénaristes eux-mêmes, qui travaillent en flux tendu sans nécessairement savoir à l'avance de combien de saisons ils disposent. À cet égard, l'ouvrage de Favard constitue un apport aux réflexions de

(voir par exemple le manuel de Sargent disponible sur openlibrary.org : Epes Winthrop Sargent, The Techniques of Photoplay, New York, Moving Picture World, 1912), mais le statut de «gourou» acquis par Syd Field - qui revient par exemple de manière obsessionnelle sur le récit de Chinatown (1974) en tentant de convaincre le lecteur que ce film estimé est réussi parce qu'il obéit au modèle qu'il préconise (alors que le film de Polanski s'en démarque sur presque tous les points) - est postérieur à l'essor du structuralisme.

7. Le degré de normativité des modèles proposés varie toutefois considérablement en fonction des auteurs : l'ouvrage de John Truby ( $L$ 'Anatomie du scénario, Paris, Nouveau Monde, 2010), qui figure dans la bibliographie de Favard, passe pour être le plus nuancé (il trouve même grâce auprès des auteurs du dossier «L'anti-manuel» du no 710 d'avril 2015 des Cahiers du cinéma). Ainsi McKee, par exemple, envisage-t-il le «schéma classique» au sein d'une tripartition comprenant par ailleurs le «minimalisme» et l' "anti-structure». Il conclut toutefois son développement par une section intitulée «L'auteur doit gagner sa vie en écrivant» dans laquelle il précise : «[L]orsque le concept s'éloigne de l'intrigue principale et descend le long du triangle vers les zones extrêmes de la mini-intrigue, de l'anti-intrigue et de la non-intrigue, le public se rétrécit» (Robert McKee, Story. Écrire un scénario pour le cinéma et la télévision, Malakoff, Armand Colin, 2017, p. 64). Notons que le terme «télévision» dans le sous-titre de l'ouvrage n'apparaît qu'à partir de cette édition récente, remplaçant «Contenu, structure, genre - les principes de l'écriture d'un scénario » de l'édition de 2001 chez Dixit (traduction littérale du titre anglais de 1997).

8. L'ouvrage qui s'appuie le plus explicitement sur la Poétique aristotélicienne et dont le titre témoigne d'un recyclage des écrits du philosophe dans le domaine de l'industrie du divertissement est celui de Ari Hiltunen, Aristotle in Hollywood : the Anatomy of Successful Storytelling, Bristol, Intellect Books, 2002. L'un des intérêts de l'ouvrage d'Hiltunen réside dans le fait qu'il envisage le récit en termes d'effets produits sur le public.

9. Fût-elle envisagée en tant que résultat d'un acte de lecture, comme chez Jean-Michel Adam qui s'inspire de la configuration narrative selon Ricœur en discutant la nécessité d'une «détermination rétrograde qui fait qu'un récit est emporté vers sa fin $(\mathrm{t}+\mathrm{n})$, organisé en fonction de cette situation finale» (Jean-Michel Adam, Le Récit, Paris, Presses universitaires de France, 1994 [1984], p. 87).

10. Voici l'annonce formulée dans l'avant-propos paradigmatique d'un manuel : «Chacun de nous à quelque chose à dire. Notre vécu se mêlant constamment à notre imaginaire, nous sommes à chaque instant créateurs en puissance. Aussi, le problème n'est-il pas de savoir quel sujet traiter, mais de savoir comment» (Jean-Marie Roth, L'Écriture de scénarios, Paris, Chiron, 2014, p. 9). Dans cet ouvrage à vocation professionnalisante, les exemples des différents types de présentation (c'est-à-dire les stades de l'écriture) sont fictifs. Il en va de même dans Dominique Parent-Altier (Approche du scénario, Paris, Armand Colin, 2005 [1997]), dont l'ouvrage a cependant le mérite de se pencher sur l'aspect (étonnamment peu traité dans les manuels) des conventions typographiques et terminologiques de l'écriture scénaristique. 
chercheuses et chercheurs qui envisagent le récit autrement que comme une totalité. Ainsi Favard opte-t-il pour des postulats méthologiques issus de la réflexion de Raphaël Baroni consacrée à la tension narrative ${ }^{11}$ (selon Baroni, «l'intrigue n'a pas besoin de reposer sur une planification 12 ») et de Marc Escola, pour qui les pratiques sérielles - et plus spécifiquement certaines fictions de l'âge classique - «obligent à substituer au "principe de causalité régressive" » qu'il observe chez Genette, Todorov ou Grivel, «ce qu' on peut bien appeler un principe d'économie prospective du récit, où le tout n'a pas autorité sur la partie13».

Le postulat théorique de Favard, qui s'appuie notamment dans le domaine de l'étude des séries TV sur les travaux de Jean-Pierre Esquenazzi, le conduit par conséquent à mettre en valeur les phénomènes de réception et de production. L'approche qui consiste à envisager les séries TV comme des «œuvres en progrès », écrites à partir de l'acceptation d'un pitch $^{14}$ sur une durée longue au cours de laquelle certains possibles sont actualisés et d'autres non, pensées pour le format exigé par les networks ou les chaînes câblées (avec des pilotes, season finales, etc.) et soumises aux aléas de la diffusion concerne inévitablement des questions relatives à la genèse de ces productions. Cependant, l'ouvrage de Favard revendique une approche résolument narratologique, enrichie ponctuellement d'une ouverture aux théories de la fiction 15 - sans pour autant aller selon nous jusqu'à prendre pleinement la mesure du rôle que jouent dans les séries étudiées, et en particulier dans Lost et Fringe auxquelles Favard confère une place nodale ${ }^{16}$, l'exacerbation de la création mondaine et l'organisation de mondes multiples 17 (ce que confirment selon nous des séries en cours telles que Stranger Things ou Westworld $\left.{ }^{18}\right)$. Cet objectif consistant à proposer «une analyse narratologique», fût-elle, comme le dit l'auteur, «agrémentée de considérations pragmatiques sur le travail d'écriture ${ }^{19}$ » (considérations dont il faut noter le degré de généralité), explique pourquoi Favard ne se préoccupe jamais de documenter le processus de genèse des textes produits par les scénaristes desdites séries, se contentant de baser ses analyses sur ce qu'il observe dans les récits filmiques (qu'il expose avec beaucoup de clarté en dépit de la complexité résultant de la déclinaison sérielle, le confort de lecture étant favorisé par un retour régulier à un petit corpus de séries relevant des «genres de l'imaginaire» - propices à une dimension méta-narrative - qui servent

11. «[...] phénomène qui survient lorsque l'interprète d'un récit est encouragé à attendre un dénouement, cette attente étant caractérisée par une anticipation teintée d'incertitude qui confère des traits passionnels à l'acte de réception» (Raphaël Baroni, La Tension narrative, Paris, Seuil, 2007, p. 18). 12. Raphaël Baroni, «Intrigue et personnages de séries évolutives», Télévision, no 7, 2016, p. 38, cité dans F. Favard, op. cit., p. 26.

13. Marc Escola, «Le clou de Tchekhov. Retours sur le principe de causalité régressive», dans La Partie et le Tout. Les moments de la lecture romanesque sous l'Ancien Régime (XVII -XVIII ${ }^{e}$ siècles), M. Escola et J.-P. Sermain (dir.), Bruxelles, Peeters, 2010, en ligne sur Atelier Fabula, cité dans F. Favard, op. cit., p. 30.

14. Voir l'intéressant encart consacré au calendrier de la genèse des pilotes aux États-Unis (F. Favard, op. cit., p. 54).

15. Par exemple, Thomas Pavel, Univers de la fiction, Paris, Seuil, 1986; Marie-Laure Ryan (dir.), Narrative across Media: The Languages of Storytelling, Lincoln, University of Nebraska Press, 2004 ; Anne Besson, D’Asimov à Tolkien, cycles et séries dans la littérature de genre, Paris, CNRS Éditions, 2004.

16. Lost représente pour Favard une «œuvre majeure, qui a marqué la télévision des années 2000 et influencé les séries qui lui ont succédé, après qu'elle a brisé les conventions de celles qui lui succédaient», tandis que Fringe est «la dernière porte-étendard [sic] d'une modalité de narration [la promesse de dénouement] qui a participé à la redéfinition des séries télévisées » (F. Favard, op. cit., p. 16-17).

17. Nous avons précisément examiné sous l'angle du worldbuilding les mêmes séries Lost et Fringe dans Cinéma, machine à mondes. Essai sur les films à univers multiples, Genève, Georg, 2014, notamment p. 169-185. La citation par Favard d'Étienne Souriau à propos de la notion de «diégèse », tirée d'un article de 1951 et non de L'Univers filmique de 1953 (la source est d'ailleurs donnée par Favard sans référence paginale), ne nous paraît pas très pertinente; le propos aurait été plus clair si l'auteur avait cité un autre passage du filmologue (sur la notion de diégèse, voir Cinéma, machine à mondes..., op. cit., p. 89-97). Pour notre part, nous ne pensons pas que les études sur les séries TV doivent se développer de manière cloisonnée par rapport aux objets traditionnels des études cinématographiques (d'autant plus aujourd'hui où les productions sérielles sont également très présentes sur grand écran).

18. Voir Alain Boillat, «Films et séries “à mondes” à l'ère numérique : le cas de Westworld», Revue de la Bibliothèque nationale de France, no 59, 2019, p. 115-123. Chez Favard, voir néanmoins la section «La saturation du monde dans Lost», pensée dans une perspective transmédiale (F. Favard, op. cit., p. 120-127).

19. F. Favard, op. cit., p. 18 (nous soulignons). 
de fil rouge à la démonstration $)^{20}$ : pas question chez lui de discuter des variantes scénaristiques qui auraient été couchées sur le papier, des documents contractuels pesant sur l'écriture ou des notes ou enregistrements issus des séances de brainstorming dans les writing rooms. À l'instar de la très grande majorité des manuels d'écriture scénaristique, qui vont jusqu'à s'appuyer sur des exemples fictifs ou non identifiés ${ }^{21}$ comme on le ferait de phrases-types dans un manuel de grammaire, les cas discutés dans l'ouvrage de Favard sont puisés dans le seul visionnement de séries, indépendamment de toute existence matérielle de sources textuelles.

Par manière de comparaison, on peut noter que Michel Chion ne procédait guère différemment dans Écrire un scénario, et ce en dépit du projet formulé en ces termes dans son introduction :

Pour chacun [des] quatre films [étudiés] est donnée une étude à la fois historique et analytique, qui met en valeur son intérêt pour l'apprentissage du scénario tout en racontant sa genèse (également intéressante, car la plupart des scénarios sont soumis à des remaniements, doivent se soumettre à des contraintes spécifiques) ${ }^{22}$.

Or, de ses contraintes relatives aux quatre exemples choisis, l'ouvrage de Chion ne nous dit en fin de compte presque rien, l'auteur se bornant à discuter, dans leurs très grandes lignes, la structure narrative ou certains aspects thématiques des quatre films sélectionnés. On peut supposer que deux des études de cas se justifient par l'existence d'un numéro de L'Avant-scène Cinéma qui leur est consacré : il s'agit de L'Intendant Sansho (Kenji Mizogushi, 1954) et de Pauline à la plage (Éric Rohmer, 1983)23. Ces publications, toutefois, ne proposent qu'un découpage d'après visionnage qui ne livre aucune information de type génétique ${ }^{24}$, contrairement à d'autres numéros de ce mensuel qui consistent exceptionnellement en la reproduction d'un document scénaristique - à l'exemple de L'Aurore scénarisé par Carl Mayer25 (un numéro qui a même été joint à une édition DVD du

20. Le terme, popularisé notamment par Anne Besson, recouvre au premier chef la science-fiction et la fantasy. Précisons que Favard est également l'auteur du Récit dans les séries de science-fiction. De Star Trek à X-Files (Paris, Armand Colin, 2018). À propos de la réflexivité du genre sciencefictionnel, voir l'ouvrage (non cité par Favard) de Richard Saint-Gelais, L'Empire du pseudo. Modernités de la science-fiction, Québec, Nota bene, 1999. Favard recourt très justement à l'exemple de l'intelligence artificielle ( «The Machine») de la série Person of Interest (S02E21-22) pour souligner la dimension «méta-sérielle» de certains motifs diégétiques (p. 39-40); il aurait pu par ailleurs faire le lien avec le «MacGuffin» hitchcockien qu'il aborde plus tard (p. 121), puisque l'individu fictif créé par The Machine s'appelle Thornhill, comme le héros de La Mort aux trousses.

21. Ainsi est-il question chez A. Cucca et P. Foti d'«un extrait du scénario d'un téléfilm» non identifié (et qui n'a sans doute pas été tourné) (op. cit., p. 33-37) et d'exemples fictifs comme Heart of Africa, destiné à expliquer la manière de développer le stade de «l'idée» (p. 99-109), ou Miami Models, «proposition-type pour une série télévisée» (p. 121-132).

22. Michel Chion, Écrire un scénario, op. cit., p. 10. Dans la réédition de 2007 qualifiée en sous-titre d' «édition définitive», cette phrase est reprise à l'identique dans la nouvelle introduction, mais porte cette fois sur quatorze films. La deuxième partie présentée comme obéissant au mode du «manuel de scénario à l'américaine» est conservée, tandis que la troisième (comprenant seulement quelques pages) portant sur les conventions d'écriture (seul aspect convoquant la matérialité des textes) a été supprimée.

23. Le cas de Rohmer est particulier en ce que les documents génétiques de ses films ne ressemblent précisément pas à un «découpage» traditionnel, ainsi qu'on peut l'observer dans les archives du cinéaste déposées à l'IMEC. On peut faire remonter sa pratique à un paradoxe qu'il formulait dans un avant-propos à la publication du scénario de ses premiers films dans la Petite Bibliothèque des Cahiers du cinéma (une collection qui mériterait une étude détaillée, y compris sur un plan génétique) : «Si j'en ai fait des films, c'est parce que je n'ai pas réussi à les écrire. Et si, d'une certaine façon, il est vrai que je les ai écrits - sous la forme même où on va les lire - c'est uniquement pour pouvoir les filmer.» (Éric Rohmer, «Avant-propos », dans Six contes moraux, Paris, Cahiers du Cinéma, 1998 [1974], p. 7.)

24. Si, pour le premier film, la mention de «découpage après montage et dialogue in extenso» en page de titre (p. 7) qui pourrait laisser penser à la traduction d'un document de production (comme ceux dont usent les traducteurs de sous-titres ou de doublages), la qualification en fin de texte, «découpage après visionnement à la table de montage» (p. 65) (réalisé par Max Tessier en collaboration avec Makoto Sato pour la traduction) signifie explicitement que tel n'est pas le cas (L'Avant-scène Cinéma, «L'Intendant Sansho», no 227, mai 1979, p. 7 et 65). Les 29 notes de bas de page consistent toutes en des explications portant sur l'histoire et la culture du Japon, jamais sur le scénario du film. Dans le second cas, le texte porte l'indication suivante : «Rédaction du découpage après visionnement plan à plan à la table de montage : Dominique Haas » (L'Avant-scène Cinéma, «Pauline à la plage», no 310, juin 1983, p. 45).

25. À propos de Mayer, voir l'une des rares monographies consacrées dans une perspective stylistique à un scénariste (qui, certes, n'est pas que scénariste) : Jürgen Kasten, Carl Mayer : Filmpoet. Ein Drehbuchautor schreibt Filmgeschichte, Berlin, Vistas, 1994. 
film de Murnau ${ }^{26}$ ) - ou qui en reprennent certains extraits là où des coupes ont été pratiquées dans le film (comme dans le scénario d'À nous la liberté du no 86 de 1968). Pour ses commentaires à propos du Port de l'angoisse, Michel Chion résume l'historique de production proposé par Bruce F. Kawin 27 dans la collection «Wisconsin/Warner Bros Screenplay Series » qui, sous la direction de Tino Balio, a publié avec des commentaires critiques approfondis plusieurs scénarios de films produits par la Warner entre 1930 et 1950 (archives déposées en 1969 au Wisconsin Center for Film and Theater); Chion mentionne Kawin succinctement sans donner de référence exacte tout en renvoyant à sa bibliographie commentée (où pourtant l'ouvrage de Kawin ne figure pas), puis, dans la réédition augmentée de 2007 dans laquelle il conserve l'historique du film de Hawks, il supprime purement et simplement la référence à Kawin, qui ne figure pas non plus en bibliographie, comme s'il importait à l'auteur d'occulter l'existence de cette édition du scénario (elle comporte pourtant un apparat critique mettant en exergue la réécriture par Hawks du scénario d'Hemingway, Furthman et Faulkner) et d'une véritable recherche génétique menée à son propos. Il est vrai que Chion prend soin de mentionner en introduction, à propos de la nature des textes «scénaristiques » qu'il produit, le point suivant : «Précisons que les textes des synopsis et des traitements sont de notre plume et sous notre responsabilité, et ne sont pas recopiés de textes préexistants 28 . » La démarche qui consiste chez Chion à mettre en exergue l' «originalité » de textes établis par lui-même d'après visionnage (c'est-à-dire dépourvus de tout statut de sources) dit combien les rivages d'une esquisse d'étude génétique sont lointains.

Autre exemple comparable, Scénarios modèles, modèles de scénarios de Francis Vanoye s'écarte lui aussi de ces rivages, même s'il le fait de manière plus concertée, l'auteur prenant la peine de se référer en introduction à ce type d'approche :

[L']étude du scénario en tant que maillon de la chaîne de production du film relève de la critique génétique, un peu comme celle des «avant-textes» pour la littérature. Perspective très riche, qui n'a encore été que peu explorée, mais qui ne sera pas la nôtre puisque nous voulons nous placer sur un plan de plus grande généralité29.

En dépit de cette manifestation d'intérêt à l'égard d'une application au médium cinématographique (et à l'écriture scénaristique en particulier) de la critique génétique, le théoricien se garde de s'en réclamer, désireux quant à lui d'en rester au niveau des principes généraux du récit filmique plutôt que d'opter pour une démarche inductive dont le point de départ serait une étude de cas. C'est pourquoi l'exposé de Vanoye se base majoritairement, à l'instar des manuels de la tradition hollywoodienne dont pourtant il entend se distinguer, non sur des documents scénaristiques, mais, de manière téléologique, sur des «modèles de scénarios [...] fournis par les films finis et par leurs transcriptions 30 ».

Pour en revenir à l'ouvrage de Favard, on observe que l'auteur ne s'appuie même pas sur des retranscriptions de scénarios mais uniquement sur le récit déduit du film. Il est vrai que dans le cas des séries discutées, qui sont relativement récentes, exclusivement nord-américaines et qui ont nécessité des moyens financiers considérables, les coulisses scénaristiques relèvent souvent du secret bien gardé - il en va de même dans le domaine des productions vidéoludiques, qui seraient elles aussi des candidats d'autant plus intéressants à une approche génétique du scénario que le produit final conserve certaines bifurcations des possibles nécessaires à l'interactivité31. À notre époque

26. L'Avant-scène Cinéma, no 148, juin 1974, a été reproduit dans un petit format joint au DVD du film édité en 2003 par Allerton Films/Carlotta Films. Ce supplément ne fait que souligner par contraste l'habituelle pauvreté en termes de documentation génétique qui a régné, au-delà de sempiternels making of ostensiblement promotionnels, dans l'édition aujourd'hui sur le déclin de DVD et Blu-ray, supports pourtant $a$ priori tout à fait adéquats à la mise à disposition de telles sources.

27. Bruce F. Kavin (dir.), To Have and Have Not, Wisconsin, University of Wisconsin Press, 1980.

28. M. Chion, op. cit., p. 10.

29. Francis Vanoye, Scénarios modèles, modèles de scénarios, Paris, Armand Colin, 2008, p. 11 (à la même page dans l'édition Nathan de 1991).

30. Ibid., p. 13.

31. Voir, à titre de prémisse, Selim Krichane, «Récit et jeux vidéo : l'exemple du scénario d'Assassin's Creed», Archipel, no 34, 2011 , p. $153-169$. 
du «temps réel» de la diffusion (thématisée dans les condensations temporelles de la série 24 Heures Chrono), la série TV semble s'accommoder difficilement d'une démarche archivistique. Enfin, le caractère collaboratif d'un processus créatif - il l'est d'autant plus à la télévision qu'au cinéma ${ }^{32}$ - souvent guidé par un principe d'improvisation ne facilite pas l'indexation de stades transitoires et la recherche de documents génétiques.

Pour aborder les coulisses de la création, Favard recourt par conséquent à un autre type de sources avec lequel il n'instaure somme toute pas un rapport fondamentalement différent de celui qu'entretiennent les communautés de fans avec les séries : il s'agit des déclarations des scénaristes, qui sont moins discutées en tant que contributions à la création d'une posture auctoriale (dans un contexte de forte légitimation des séries TV américaines) ${ }^{33}$ qu'à l'aune des informations qu'elles trahissent sur la manière dont ces auteurs conçoivent le récit sériel, ou sur les contraintes auxquelles ils ont été confrontés. Il en va par exemple ainsi d'un commentaire de Ronald D. Moore, showrunner, sur Battlestar Galactica, disponible dans un podcast et repris sur la piste audio de l'édition DVD de la série 34 . Dans une démarche explicite de réhabilitation de la série Babylon 5 (1993-1998), Favard convoque à titre exceptionnel des données relatives à la genèse de l'œuvre :

La légende veut que J. Michael Straczynski, le créateur de la série, ait planifié les cinq saisons à l'avance. C'est sans doute $v r a i$, et, pour autant qu'ils soient dispersés, de nombreux documents permettent de mener une analyse génétique de la création de la série. [...] Si l'on en croit les archives disponibles sur le site JMSNews, Straczynski a commencé à chercher le soutien de la communauté de fans de science-fiction dès novembre 199135 .
Favard formule les précautions méthodologiques d'usage mais n'en témoigne pas moins d'un refus de se pencher véritablement sur des sources. Elles sont il est vrai excessivement foisonnantes, et l'examen de celles-ci excéderait le propos de l'auteur qui consiste surtout à se demander, comme il le précise dans le titre d'un encadré, si Straczynski est un «précurseur des showrunners d'aujourd'hui» (la réflexion sur la promesse d'un dénouement, on le voit, ne prémunit pas l'auteur contre une conception téléologique de l'histoire des pratiques télévisuelles). Ce n'est pas un hasard si l'idée d'une documentation de la genèse concerne une série antérieure à celles qui forment le cœur du corpus étudié : l'accent mis sur le contemporain - argument promotionnel évident pour l'ouvrage (par exemple, Game of throne mentionné en quatrième de couverture n'occupe en fait qu'une petite place dans l'étude) en regard de l'oubli souvent rapide auquel sont condamnées ces productions qui se relaient dans le flux constant de la télédiffusion constitue un obstacle au recul indispensable à l'approche génétique, laquelle s'inscrit le plus souvent, sur le modèle premier des manuscrits autographes, dans le prolongement d'une démarche de conservation du patrimoine culturel (alors qu'ici le pitch s'apparente à un brevet industriel). Ce qui nous paraît quelque peu problématique dans la manière dont Favard tire parti des déclarations de quelques célèbres showrunners; c'est qu'il reprend à son compte des notions proposées par ceux-ci. Dans le cas de Straczynski, il s'agit de l' "holographic storytelling» mentionné par le scénariste sur son blog en 1993 (avec une référence très discutable et non discutée par Favard à la structure épique prônée par Bertolt Brecht), qui donne son titre à la section correspondante d'Écrire une série $T V$ tout en ne servant guère son propos (par ailleurs très pertinent ici, où il s'agit de

32. C'est d'ailleurs sans doute parce que l'étape de l'écriture n'est que peu «auteurisée» que les études cinématographiques rechignent à se pencher sur elle. Rappelons toutefois l'étude significativement parue dans le numéro de la présente revue dédié à l'écriture collaborative : Adrien Gaillard et Julien Meyer, «Jean Aurenche, Pierre Bost et Claude Autant-Lara, auteurs de Douce. Genèse d'une pratique scénaristique », Genesis, no 41, 2015, p. 96-100, en ligne sur openedition.org.

33. De sorte que nous faisons nôtres les postulats méthodologiques exposés dans l'éditorial du numéro de Décadrages comprenant un dossier intitulé «Séries télévisées contemporaines » et dirigé par Mireille Berton : «En entendant apporter une pierre à un édifice plus que prospère, nous ne souhaitons pas pour autant reproduire ici les tics de langage propres aux discours de la distinction qui voient dans les séries télévisées une forme d'anoblissement culturel de la télévision et de leurs usagers. Nous passerons également outre les justifications d'usage qui se défendent de parler d'un objet longtemps déprécié par les élites intellectuelles et sociales » (Décadrages, no 32-33, printemps 2016, p. 4).

34. F. Favard, op. cit., p. 92-93.

35. Ibid., p. 135 (nous soulignons). 
discuter à l'échelle d'un épisode, d'une saison ou d'une série «complète» les « divers procédés visant à faire résonner les éléments narratifs entre eux »36, et notamment à filer certains motifs symboliques). Au titre des notions «définies» par les showrunners eux-mêmes et reprises par Favard, on peut citer en outre celle de «mythalone» (p. 141-142) utilisée par les scénaristes Pinkner et Wyman sur le blog de leur série Fringe, voire celle aux contours flous de «mythologie» devenue courante auprès des fans : selon nous, même si la théorie ne doit pas se fermer au discours de ce type de communautés (souvent fort érudites, comme l'a noté Jenkins à propos de la «culture de la convergence»), des catégories descriptives à vocation critique gagnent à être élaborées en dehors du champ de l'instance de production dont émane pour le fan une «parole d'évangile» (ce qui appartient au «canon» ou non, voire, pour Lost, ce qui relève de la croyance). Les affirmations des auteurs sont certes précieuses pour commenter un processus génétique (pour autant qu'elles puissent être contextualisées au sein d'un réseau discursif), mais elles le sont moins pour faire évoluer une tradition théorique (en l'occurrence ici la narratologie) vers une prise en compte plus adéquate des phénomènes sériels.

Les informations relatives aux coulisses de la création égrainées chez Florent Favard permettent au lecteur de se faire une idée plus précise d'un des métiers de l'audiovisuel (scénariste de série TV), dans la tradition de certains ouvrages d'histoire du cinéma qui documentent des techniques spécifiques. L'auteur ne fait toutefois aucun cas de l'objet de l'acte d'écriture affiché dès le titre de l'ouvrage : le scénario en tant que texte(s). Comme nous l'avons montré ici à partir de quelques exemples de publications, il en va de même de la quasi-intégralité des publications dédiées au scénario ou au récit filmique - le premier étant en général confondu avec le second, alors que nous sommes d'avis, à l'instar de Jean-Michel Bertrand, qu' «il convient [...] de différencier le document écrit qui, en amont du film, donnera lieu au découpage et ce que l'on appelle plus couramment le scénario tel qu'il apparaît dans le mouvement de la vision $^{37}$ ». En effet, si certains manuels ou ouvrages à vocation «technique» intègrent un bref extrait ou quelques pages d'une source scénaristique immédiatement identifiable en tant que telle sur le plan typographique ${ }^{38}$, elle y est en général présentée comme étant le scénario du film, comme si le document, qui a le plus souvent été déposé auprès d'une instance de gestion des droits ou distribué à l'équipe du film, n'était pas le résultat de plusieurs états du texte, sur le plan tant de sa forme (le plus souvent, seules les continuités dialoguées sont conservées) que de sa genèse. Or, il nous semble qu'une réflexion sur l'écriture scénaristique pourrait tirer parti d'une étude, à partir de cas concrets, d'alternatives non retenues ou de variantes ponctuelles écartées. Certes, le scénario, «objet éphémère qui, une fois le film tourné et mis en boîte, disparaît de la circulation 39 » ${ }^{2}$ 'approche pasolinienne consistant à l'appréhender comme une «structure tendant à être une autre structure» n'en atténuait que partiellement l'évanescence ${ }^{40}$-, n'a souvent pas été conservé aux différents stades de son écriture. Certaines recherches

36. Ibid., p. 137.

37. Jean-Michel Bertrand «Films à scénario, films à histoire», dans R. Monnier et A. Roche (dir.), Territoires du scénario, Dijon, Centre G. Bachelard, 2006, p. 97.

38. Il s'agit notamment d'ouvrages (anglo-saxons) présentant une certaine fibre historique - l'histoire des pratiques scénaristiques au cinéma restant très largement à faire -, à l'instar de Steven Price, A History of Screenwriting, Houndmills, Basingstoke, Hampshire/New York, Palgrave Macmillan, 2013. On peut en outre citer les extraits inédits des scénarios de Twelve Monkeys (1995) et Shooting Fish (1997) dans Philip Parker, The Art of Science \& Screenwriting (Exeter : Intellect Books, 1999 [1998], p. 136-148 et 187-189), ou les nombreux extraits cités dans Kevin Alexander Boon, Script Culture and the American Screenplay, Detroit, Wayne State University Press, 2008.

39. Christian Biegalski, Scénarios : modes d'emploi, Paris, Dixit, 2003, p. 269. Notons que chez Biegalski, dont l'ouvrage se veut moins dogmatique et plus sensible à la dimension génétique que beaucoup d'autres manuels, la conception du récit est en quelque sorte désolidarisée de l'écriture proprement dite, posée comme antérieure : «Bien avant le film, bien avant le scénario lui-même (dans sa première ou son ultime version prête à tourner), il existe en amont de ces objets, connus et reconnus, tout un processus d'élaboration, de conception, de gestation beaucoup plus complexe et subtile que nous appellerons génériquement la scénarisation, c'est-à-dire tout un travail de préparation [...] si l'on veut, un jour, se lancer dans l'écriture d'un scénario » (ibid., p. 13). Il nous paraît au contraire que «ces objets», dans leur pluralité, ne sont guère connus et encore moins reconnus, et que le travail de gestation dont il est question ici n'est pas sans laisser, lui aussi, des traces écrites qui appartiennent précisément auxdits «objets».

40. Pier Paolo Pasolini, L’Expérience hérétique. Langue et cinéma, Paris, Payot, 1976, ch. IX. 
basées sur l'exploitation de fonds d'archives se prêtant à une telle approche ont toutefois ouvert la voie à l'analyse de documents de production dans le cadre de l'établissement du dossier génétique d'un scénario de film ${ }^{41}$, à commencer par les études figurant au sommaire du numéro 28 de Genesis paru en 2007, panorama qui représente un incontestable jalon dans le domaine mais qui n'en appelle pas moins à un examen plus approfondi des textes scénaristiques sur un plan linguistique et à un élargissement du corpus au-delà d'études de cas centrées sur des figures auteurisées dont la pratique s'écarte de la norme. Parallèlement à certaines monographies qui envisagent la carrière de cinéastes en examinant des archives de production ${ }^{42}$ et après la rigoureuse édition critique du scénario de La Règle du jeu de Jean Renoir par Curchod et Faulkner ${ }^{43}$ - à notre connaissance, un apax en la matière dans le domaine francophone ${ }^{44}-$, la monumentale étude de Jean-Louis Jeannelle 45 consacrée aux différents projets d'adaptation de La Condition humaine fait indubitablement office de modèle. Si l'intérêt méthodologique d'une approche consistant à examiner précisément des scénarios n'ayant en fin de compte pas conduit à la réalisation d'un film est indéniable 46 , il peut cependant en résulter une forme de dépréciation de recherches similaires qui porteraient quant à elles sur des scénarios de films existants, au motif que le document perdrait de son intérêt une fois le film réalisé et diffusé. Selon nous, les textes scénaristiques méritent d'être étudiés indépendamment de leur statut d'œuvre (ils résultent de «pratiques», «techniques » conduites collectivement et propres à des époques et corpus donnés) et demandent à ce que soit davantage convoquée la tradition analytique développée dans le champ des études cinématographiques (puisque dans de tels cas, l'un des comparants est le film même). Dans une telle perspective interdisciplinaire, le champ des possibles de la recherche s'avère plus vaste encore que celui des récits promulgués par les manuels, même si la faisabilité et la portée des études de cas demeurent tributaires de l'existence et de la richesse de fonds d'archives (et, pour des scénarios récents, de l'accès aux strates successives de documents nativement numériques). Le séminaire «Genèses cinématographiques », conduit conjointement à l'ENS par Daniel Ferrer et Jean-Loup Bourget puis François Thomas, a constitué à n'en pas douter l'un des principaux lieux de stimulation de ce type de recherches. À titre personnel, nous avons par ailleurs codirigé à l'Université de Lausanne deux projets collectifs soutenus par le Fonds national suisse de la recherche scientifique auxquels sont associées des thèses en cours ${ }^{47}$. L'approche affinée dans le cadre de ces projets a été mise en œuvre dans une perspective d'étude du processus de l'adaptation dans un ouvrage récent que nous avons sous-titré «essai de génétique scénaristique», et qui inaugure chez l'éditeur genevois Droz une nouvelle collection («Ciné courant») destinée à accueillir ce type d'approches. Il s'agit d'une monographie qui se consacre modestement à un unique film (En cas de malheur, 1958) envisagé au travers du palimpseste constitué des états et variantes scénaristiques que nous avons étudiés à partir des pièces (variantes scénaristiques, documents juridiques,

\footnotetext{
41. Pour un état des lieux, nous renvoyant au ch. III («La critique génétique appliquée au scénario») de notre ouvrage En cas de malheur, de Simenon à Autant-Lara. Essai de génétique scénaristique, Genève, Droz, 2020, p. 147-185.

42. Voir, à l'échelle de la filmographie complète d'un réalisateur, la récente monographie de Jean-Pierre Bleys, Claude Autant-Lara, Arles, Actes Sud/ Institut Lumière, 2018 (à propos de certaines limites méthodologiques de cette recherche, voir le compte rendu que nous avons fait paraître dans 1895. Revue de l'association française de recherche sur l'histoire du cinéma, no 88, 2019, p. 167-176).

43. Olivier Curchod et Christopher Faulkner, La Règle du jeu - scénario original de Jean Renoir, Paris, Nathan Cinéma, 1999.

44. L'ouvrage de Gérard Leblanc et Brigitte Devismes, Le Double Scénario chez Fritz Lang (Paris, Armand Colin, 1990) ne manque pas d'intérêt pour examiner la préparation de la mise en scène par Lang, mais il néglige étonnamment les textes scénaristiques proprement dits.

45. Jean-Louis Jeannelle, Films sans images. Une histoire des scénarios non réalisés de La Comédie humaine, Paris, Seuil, 2015.

46. Voir l'Anthologie du cinéma invisible de Christian Janicot (Paris, Éditions Jean-Michel Place/Arte, 1995), et à propos de celle-ci Carole Aurouet, «Anthologie du cinéma invisible : genèse - conception - réception », dans M. Brangé et J.-L. Jeannelle (dir.), Films à lire. Des scénarios et des livres, Bruxelles, Impressions Nouvelles, 2019, p. 137-158.

47. Il s'agit des projets suivants : «Discours du scénario : étude historique et génétique des adaptations cinématographiques de Stendhal (2013-2017); «Personnage et vedettariat au prisme du genre : étude de la fabrique des représentations cinématographiques » (2016-2020). Voir à leur propos le site http://wp.unil.ch/cinematheque-unil/ et l'ouvrage d'Alain Boillat et Gilles Philippe (dir.), L'Adaptation - Des livres aux scénarios. Approche interdisciplinaire des archives du cinéma français (1930-1960), Bruxelles, Les Impressions Nouvelles, 2018.
} 
correspondance, notes diverses, etc.) issues du fonds composé des archives personnelles du cinéaste Claude Autant-Lara déposées à la Cinémathèque suisse ${ }^{48}$. Il faut noter par ailleurs que «l'avant-texte» d'un film ne se réduit pas à l'étape de l'écriture : Emmanuelle Meunier a étudié la genèse du Casanova de Fellini en complétant la reconstitution du contexte de production et la comparaison entre trois versions du scénario (dont l'une publiée chez Einaudi) par l'examen de suppressions effectuées au tournage et de modifications intervenues à l'étape de la postproduction, notant en particulier une tendance à substituer la voix in du personnage par la voix over du narrateur 49 . En retournant ainsi aux alternatives non retenues, la chercheuse acquiert une connaissance enrichie de l'œuvre filmique et du rapport qu'elle instaure avec l'œuvre adaptée. En faisant de ces documents jusqu'ici passablement occultés que sont synopsis, séquenciers, traitements, continuités dialoguées et découpages techniques des objets d'étude à part entière, nous pensons que l'approche génétique des scénarios de films (et pourquoi pas de séries TV) pourrait fournir une contribution importante tant à l'histoire du cinéma qu'à la critique génétique.

48. Alain Boillat, En cas de malheur, de Simenon à Autant-Lara, op. cit.

49. Emmanuelle Meunier, Fellini et Casanova, Paris, Presses universitaires de Vincennes, 2014. L'auteure utilise en fait non le terme de «voix over» mais celui de « voix-off»; nous ne reviendrons pas ici sur l'intérêt à privilégier le premier - nous le commentons largement dans Du bonimenteur à la voix-over (Lausanne, Antipodes, 2007).

Professeur d'histoire et d'esthétique du cinéma à l'Université de Lausanne, ALAIN BoILLAT dirige le Centre d'études cinématographiques (CEC). Ses recherches ont pour objet l'étude historique, génétique et narratologique du scénario (En cas de malheur, de Simenon à Autant-Lara. Essai de génétique scénaristique, Droz, 2020), le rôle de la voix et de l'oralité dans les dispositifs audiovisuels (Du bonimenteur à la voix-over, 2007), les imaginaires de la technologie, la science-fiction, la sérialité et la fiction au cinéma et en bande dessinée (La Fiction au cinéma, L’Harmattan, 2001 ; Cinéma, machine à mondes. Essai sur les films à univers multiples, Georg, 2014 ; Star Wars, un monde en expansion, ActuSF, 2016). Il a aussi codirigé les ouvrages suivants : Dubbing. La Traduction audiovisuelle (Schüren, 2014), Dialogues avec le cinéma. Approches de l'oralité cinématographique (Nota Bene, 2016), BD-US : les comics vus par l'Europe (Infolio, 2016), Case, strip, action! Les feuilletons en bandes dessinées dans les magazines pour la jeunesse (1946-1959) (Infolio, 2016), Je suis ton père. Origines et héritages d'une saga intergalactique (2018), L'Adaptation. Des livres aux scénarios (Impressions Nouvelles, 2018) et Loin des yeux... le cinéma. De la téléphonie à Internet : imaginaires médiatiques des télécommunications et de la surveillance (L'Âge d'Homme, 2019).

alain.boillat@unil.ch 